\title{
Resolution complexity of perfect matching principles for sparse graphs*
}

\author{
Dmitry Itsykson $^{\dagger 1}$, Mikhail Slabodkin ${ }^{\ddagger 2}$, and Dmitry Sokolov ${ }^{\S 1}$ \\ ${ }^{1}$ Steklov Institute of Mathematics at St.Petersburg \\ ${ }^{2}$ St. Petersburg Academic University
}

July 23, 2014

\begin{abstract}
The resolution complexity of the perfect matching principle was studied by Razborov [Raz04], who developed a technique for proving its lower bounds for dense graphs. We construct a a constant degree bipartite graph $G_{n}$ such that the resolution complexity of the perfect matching principle for $G_{n}$ is $2^{\Omega(n)}$, where $n$ is the number of vertices in $G_{n}$. This lower bound matches with the upper bound $2^{O(n)}$ up to an application of a polynomial. Our result implies the $2^{\Omega(n)}$ lower bounds for the complete graph $K_{n}$ and the complete bipartite graph $K_{n, O(n)}$ that improve the lower bounds followed from [Raz04]. Our results also implies the well-known exponential lower bounds on the resolution complexity of the pigeonhole principle, the functional pigeonhole principle and the pigeonhole principle over a graph.

We also prove the following corollary. For every natural number $d$, for every $n$ large enough, for every function $h:\{1,2, \ldots, n\} \rightarrow\{1,2, \ldots, d\}$, we construct a graph with $n$ vertices that has the following properties. There exists a constant $D$ such that the degree of the $i$-th vertex is at least $h(i)$ and at most $D$, and it is impossible to make all degrees equal to $h(i)$ by removing the graph's edges. Moreover, any proof of this statement in the resolution proof system has size $2^{\Omega(n)}$. This result implies well-known exponential lower bounds on the Tseitin formulas as well as new results: for example, the same property of a complete graph.
\end{abstract}

\section{Introduction}

The resolution proof system is one of the simplest and well-studied proof systems. There are well known methods of proving lower and upper bounds on the complexity of several

*The research is partially supported by the RFBR grant 14-01-00545, by the President's grant MK2813.2014.1 and by the Government of the Russia (grant 14.Z50.31.0030).

†dmitrits@pdmi.ras.ru

${ }_{\ddagger}^{\ddagger}$ slabodkinm@gmail.com

§sokolov.dmt@gmail.com 
types of formulas. However, there are no known universal methods to determine an asymptotic resolution complexity of a given family of formulas. We say that a family of unsatisfiable CNF formulas $F_{n}$ is weaker than a family of unsatisfiable formulas $H_{n}$ if every clause of $H_{n}$ is an implication of a constant number of clauses of $F_{n}$. Since the resolution proof system is implication complete, the size of any resolution proof of $H_{n}$ is at least the size of the minimal resolution proof of $F_{n}$. Thus it is interesting to prove lower bounds for for fomulas as weak as possible.

CNF formulas $\mathrm{PHP}_{n}^{m}$ encode the pigeonhole principle; $\mathrm{PHP}_{n}^{m}$ states that it is possible to put $m$ pigeons into $n$ holes in such a way that every pigeon is contained in at least one hole and every hole contains at most one pigeon. $\mathrm{PHP}_{n}^{m}$ depends on variables $p_{i, j}$ for $i \in[m]$ and $j \in[n]$ and $p_{i, j}=1$ iff the $i$-th pigeon is in the $j$-th hole. $\mathrm{PHP}_{n}^{m}$ is unsatisfiable iff $m>n$. Haken [Hak85] proved the lower bound $2^{\Omega(n)}$ on the resolution complexity of $\mathrm{PHP}_{n}^{n+1}$. Raz [Raz01a] proved the lower bound $2^{n^{\epsilon}}$ on the resolution complexity of $\mathrm{PHP}_{n}^{m}$ for some positive constant $\epsilon$ and arbitrary $m>n$. This lower bound was simplified and improved to $2^{\Omega\left(n^{1 / 3}\right)}$ by Razborov [Raz01b].

Urquhart [Urq03] and Ben-Sasson, and Wigderson [BSW01] consider formulas $G-\mathrm{PHP}_{m}^{n}$ that are defined by a bipartite graph $G$; the first part of $G$ corresponds to pigeons and consists of $m$ vertices, and the second part corresponds to holes and consists of $n$ vertices. Every pigeon must be contained in one of adjacent holes. Formulas $G-\mathrm{PHP}_{n}^{m}$ may be obtained from $\mathrm{PHP}_{n}^{m}$ by substituting variables which do not have corresponding edges in $G$ with zeroes. The paper [BSW01] presents the lower bound $2^{\Omega(n)}$ for formulas $G-\mathrm{PHP}_{n}^{m}$ where $m=O(n)$ and $G$ is a bipartite constant degree expander.

Razborov [Raz03] considers a so called functional pigeonhole principle $\mathrm{FPHP}_{n}^{m}$ that is a weakening of $\mathrm{PHP}_{n}^{m}$; the formula $\mathrm{FPHP}_{n}^{m}$ is the conjunction of $\mathrm{PHP}_{n}^{m}$ and additional conditions stating that every pigeon is contained in at most one hole. Razborov proved a lower bound $2^{\Omega\left(\frac{n}{(\log m)^{2}}\right)}$ for $\mathrm{FPHP}_{n}^{m}$ that implies a lower bound $2^{\Omega\left(n^{1 / 3}\right)}$ depending only on $n$.

Let for every graph $G$ a formula $\mathrm{PMP}_{G}$ (from the Perfect Matching Principle) encode that $G$ has a perfect matching. Variables of $\mathrm{PMP}_{G}$ correspond to edges, and for every vertex of $G$ exactly one incident edge has value 1. Razborov [Raz04] proved that if $G$ has no perfect matchings, then the resolution complexity of $\mathrm{PMP}_{G}$ is at least $2^{\frac{\delta(G)}{\log ^{2} n}}$, where $\delta(G)$ is the minimal degree of the graph and $n$ is the number of vertices.

Alekhnovich [Ale04] and Dantchev and Riis [DR01] consider the graphs of the chessboard $2 n \times 2 n$ with two opposite corners removed. The perfect matching principle for such graphs is equivalent to the possibility to tile such chessboards with domino. The strongest lower bound $2^{\Omega(n)}$ was proved in [DR01] and this lower bound is polynomially connected with the upper bound $2^{O(n)}$. We note that the number of variables is $\Theta\left(n^{2}\right)$.

Our results For all $n$ and all $m \in[n+1, O(n)]$ we give an example of a bipartite graph $G_{m, n}$ with $m$ and $n$ vertices in its parts such that all degrees are bounded by a constant and the resolution complexity of $\mathrm{PMP}_{G_{m, n}}$ is $2^{\Omega(n)}$. The number of variables in such formulas is $O(n)$, therefore the lower bound matches (up to an application of a polynomial) the trivial upper bound $2^{O(n)}$ that holds for every formula with $O(n)$ variables. This is the first lower bound for perfect matching principle that is exponential in the number of variables. In particular, our results imply that the resolution complexity 
of $\mathrm{PMP}_{K_{m, n}}$ is $2^{\Omega(n)}$, where $K_{m, n}$ is the complete bipartite graph and $m=O(n)$. And this lower bound improves the lower bound $2^{\Omega\left(n / \log ^{2} n\right)}$ that follows from [Raz04] and matches (up to a polynomial application) the upper bound $n 2^{n}$ that follows from the upper bound for $\mathrm{PHP}_{n}^{n+1}$ [SB97]. Our result implies the lower bound $2^{\Omega(n)}$ on the resolution complexity of $\mathrm{PMP}_{K_{n}}$, where $K_{n}$ is a complete graph on $n$ vertices, and it is also better than the lower bound $2^{\Omega\left(n / \log ^{2} n\right)}$ that follows from [Raz04]. We note that $P M P_{G_{m, n}}$ is weaker than $G_{m, n}-\mathrm{PHP}_{n}^{m}, \mathrm{PHP}_{n}^{m}$ and $\mathrm{FPHP}_{n}^{m}$, therefore our lower bound implies the same lower bound for $G_{m, n}-\mathrm{PHP}_{n}^{m}, \mathrm{PHP}_{n}^{m}$ and $\mathrm{FPHP}_{n}^{m}$. To put it more precisely, we we prove the following theorem:

Theorem 1.1. Let $G$ be a bipartite graph with parts $X$ and $Y$ such that the folowing holds:

1. $G$ is a $(r, c)$-boundary expander; i.e. for all $A \subseteq X$, if $|A| \leq r$ then $|\delta(A)| \geq c|A|$, where $\delta(A)$ is the set of all vertices in $Y$ that are connected with exactly one vertex in $A$;

2. There is a matching in $G$ that covers all vertices from $Y$.

Then the width of all resolution proofs of $\mathrm{PMP}_{G}$ is at least $c r / 2$. If additionally degrees of all vertices are at most $D$, then (using [BSW01] we get that) the size of any resolution proof of $P H P_{G}$ is at least $2^{\Omega\left(\frac{(c r / 2-D)^{2}}{n}\right)}$, where $n$ is the number of edges in $G$.

The condition that $G$ has a matching covering all vertices from $Y$ cannot be removed for free since for every $(r, c)$-boundary expander it is possible to add one vertex to $X$ and $\lceil c\rceil$ vertices to $Y$ such that the new vertex in $X$ is connected with all new vertices in $Y$. The resulting graph is also $(r, c)$-boundary expander but the resulting formula will contain unsatisfiable subformula that depends on $\lceil c\rceil$ variables, hence it can be refuted with width $\lceil c\rceil$. We do not know whether it is possible to replace the second condition in the theorem by a weaker condition.

To estimate the width we use the method introduced by Ben-Sasson and Wigderson in [BSW01]. However, we use a non-standard notion of a semantic implication and a non-standard measure on the set of clauses.

An example of a graph that suits the conditions of Theorem 1.1 can be constructed from every lossless expander by removing vertices of high degrees as it was shown in [IS11], and by adding a matching that covers all vertices from $Y$. For example, we can use the explicit construction of lossless expanders from [MCW02] (or the randomized construction [HLW06]).

Theorem 1.1 implies a more general theorem:

Theorem 1.2. For graph $G(V, E)$ and function $h: V \rightarrow\{1,2, \ldots, d\}$ we define a formula $\Psi_{G}^{(h)}$, that code that $G$ has a subgraph $H$ such that for all $v$ in $H$ the degree of $v$ equals $h(v)$. For any $d \in \mathbb{N}$, there exists $D \in \mathbb{N}$ that for all $n$ large enough and every function $h: V \rightarrow\{1,2, \ldots, d\}$, where $|V|=n$, there exists graph $G(V, E)$ with degrees of vertices at most $D$ such that the formula $\Psi_{G}^{(h)}$ is unsatisfiable and the size of any resolution proof of $\Psi_{G}^{(h)}$ is at least $2^{\Omega(n)}$. 
If $h$ maps $V$ to $\{1,2\}$, then $\Psi_{G}^{(h)}$ is weaker than Tseitin formulas based on graph $G$. Thus our result implies the lower bound $2^{\Omega(n)}$ on the resolution complexity of Tseitin formulas that was proved in [Urq87].

\section{Preliminaries}

We consider simple graphs without loops and multiple edges. The graph $G$ is called bipartite if its vertices can be divided into two disjoint parts $X$ and $Y$ in such a way that any edge is incident to one vertex from $X$ and one vertex from $Y$. We denote $G(X, Y, E)$ a bipartite graph with parts $X$ and $Y$ and set of edges $E$. A matching in a graph $G(V, E)$ is such a set of edges $E^{\prime} \subseteq E$ that any vertex $v \in V$ has at most one incident edge from $E^{\prime}$. A matching $E^{\prime}$ covers a vertex $v$ if there exists $e \in E^{\prime}$ that is incident to $v$. A perfect matching is a matching that covers all vertices of $G$. For a bipartite graph $G(X, Y, E)$ and a set $A \subseteq X$ we denote $\Gamma(A)$ a set of all neighbors of vertices from $A$.

Lemma 2.1 (Hall). Consider such a bipartite graph $G(X, Y, E)$ that for some $A \subseteq X$ for all $B \subseteq A$ the following inequality holds: $\Gamma(B) \geq|B|$. Then there is a matching that covers all vertices from $A$.

For a CNF formula $\varphi$ a proof of its unsatisfiability in the resolution proof system is a sequence of clauses with the following properties: the last clause is an empty clause (we denote it by $\square$ ); any other clause is either a clause of initial formula $\varphi$ or can be obtained from previous ones by the resolution rule. The resolution rule admits to infer a clause $(B \vee C)$ from clauses $(x \vee B)$ and $\neg x \vee C$. The size of a resolutional proof is the number of clauses in it.

In [BSW01] E. Ben-Sasson and A. Wigderson introduced a notion of formula width. A width of a clause is a number of literals contained it it. For a $k$-CNF formula $\varphi$ a width of $\varphi$ is a maximum width of clauses of $\varphi$. A width of a resolution proof is a width of the largest clause used in it.

Theorem 2.1 ([BSW01]). For any $k$-CNF unsatisfiable formula $\varphi$ the size of resolution proof is at least $2^{\Omega\left(\frac{(w-k)^{2}}{n}\right)}$, where $w$ is a minimal width of a resolutional proof and $n$ is a number of variables used in $\varphi$.

Lemma 2.2. Let $\phi$ be a formula that is obtained from unsatisfiable formula $\psi$ by a substitution of several variables. Then $\phi$ is unsatisfiable and the size of the minimal resolution proof of $\psi$ is at least the size of the minimal resolution proof of $\phi$.

\section{Subgraph extraction}

\subsection{Existence of a perfect matching}

For an undirected graph $G(V, E)$ we construct a formula $\mathrm{PMP}_{G}$ that encodes that $G$ has a perfect matching. We assign a binary variable $x_{e}$ for all $e \in E \cdot \mathrm{PMP}_{G}$ is the conjunction of the following conditions: for all $v \in V$ exactly one edge that incident to $v$ has value 1 . Such conditions can be written as the conjunction of the statement that at 
least one edge takes value 1: $\bigvee_{(v, u) \in E} x_{(v, u)}$ and the statement that for any pair of edges $e_{1}, e_{2}$ incident to $v$ at most one of them takes value $1: \neg x_{e_{1}} \vee \neg x_{e_{2}}$.

Note that if degrees of all vertices are at most $D$, then $\mathrm{PMP}_{G}$ is a $D$-CNF formula.

In this section we prove the following theorem:

Theorem 3.1. There exists a constant $D$ such that for all $C$ that for all $n$ large enough and for all $m \in[n+1, C n]$ it is possible to construct in polynomial in $n$ time such bipartite graph $G(V, E)$ with $m$ and $n$ vertices in parts that all degrees are at most $D$ and the formula $\mathrm{PMP}_{G}$ is unsatisfiable and the size of any resolution proof of $\mathrm{PMP}_{G}$ is at least $2^{\Omega(n)}$.

Definition 3.1. A bipartite graph $G(X, Y, E)$ is $(r, c)$-boundary expander if for any set $A \subseteq X$ such that $|A| \leq r$ the following inequality holds $|\delta(A)| \geq c|A|$, where $\delta(A)$ denotes the set of all such vertices in $Y$ that are connected with the set $A$ by the unique edge.

Lemma 3.1. Let bipartite graph $G(X, Y, E)$ have two matchings, the first one covers all vertices from $Y$ and the second covers all vertices from $A \subseteq X$. Then there exists a matching in $G$ that covers $A$ and $Y$ simultaneously.

Proof. Let $L$ denote the matching that covers all vertices from the set $A$ and let $F$ be a matching that covers all vertices from $Y$. We prove that if $F$ does not cover all vertices from $A$, then one may construct a matching $F^{\prime}$ that covers more vertices of $A$ than $F$ and also covers all vertices from $Y$. Therefore there is such a matching that covers $A$ and $Y$.

Consider some vertex $v_{1} \in A$ that is not covered by $F$ and such path $v_{1}, u_{1}, v_{2}, u_{2}, \ldots, u_{k-1}, v_{k}$ that $\left(v_{i}, u_{i}\right) \in L,\left(u_{i}, v_{i+1}\right) \in F$ and $v_{1}, v_{2}, \ldots, v_{k-1} \in A$ and $v_{k} \notin A$.

For any fixed $v_{1} \in A$ such a path can be constructed deterministically: starting at vertex $v_{1}$ the edges of the path belong to alternating matchings $L$ and $F$. For every vertex from $X$ at most one of outgoing edges belongs to $L$. For every vertex from $Y$ exactly one of outgoing edges belongs to $F$. The path can't become a cycle because $v_{1}$ has no incident edges from $F$, therefore the constructed path will lead to some vertex $v_{k} \notin A$.

Let matching $F^{\prime}$ be constructed from $F$ by removing all edges $\left(v_{i}, v_{i+1}\right)$ and adding edges $\left(u_{i}, v_{i}\right)$ for $1 \leq i<k$. Now $F^{\prime}$ covers all $Y$ and covers one additional vertex of $A$ in comparison with $F$.

Lemma 3.2. Let $G(X, Y, E)$ be a bipartite $(r, d, c)$-boundary expander with $c>2$ and $|X|>|Y|$. Let $G$ have a matching that covers all vertices from the part $Y$. Then the formula $\mathrm{PMP}_{H}$ is unsatisfiable and the width of its resolution refutation is at least $\mathrm{cr} / 2$.

Proof. Parts $X$ and $Y$ have different number of vertices, hence there are no perfect matchings in $G$ and $\mathrm{PMP}_{G}$ is unsatisfiable.

We call an assignment to variables of $\mathrm{PMP}_{G}$ proper if for every vertex $v$ at most one edge incident to $v$ has value 1 . For some subset $S \subseteq V$ and for a clause $C$ we say that $S$ properly implies $C$ if any proper assignment that satisfies all constraints in vertices from $S$, also satisfies $C$. We denote it as $S \vdash C$.

Now we define a measure on clauses from a resolution refutation of $\mathrm{PMP}_{G}: \mu(C)=$ $\min \{|S \cap X| \mid S \vdash C\}$. 
The measure $\mu$ has the following properties:

1) The measure of any clause from $\mathrm{PMP}_{G}$ equals 0 or 1 .

2) Semiadditivity: $\mu(C) \leq \mu\left(C_{1}\right)+\mu\left(C_{2}\right)$, if $C$ is obtained by applying of resolution rule to $C_{1}$ and $C_{2}$.

Let $S_{1} \vdash C_{1},\left|S_{1} \cap X\right|=\mu\left(C_{1}\right)$ and $S_{2} \vdash C_{2},\left|S_{2} \cap X\right|=\mu\left(C_{2}\right)$. Hence $S_{1} \cup S_{2} \vdash C_{1}$ and $S_{1} \cup S_{2} \vdash C_{2}$, so $S_{1} \cup S_{2} \vdash C$, therefore $\mu(C) \leq\left|S_{1} \cap X\right|+\left|S_{2} \cap X\right|=\mu\left(C_{1}\right)+\mu\left(C_{2}\right)$.

3) The measure of the empty clause $\square$ is more than $r$.

Let $\mu(\square) \leq r$, then there is such $S \subseteq V$ that $S \vdash \square$ and $|S \cap X| \leq r$. For all $A \subseteq S \cap X$ the following holds $|\Gamma(A)| \geq|\delta(A)| \geq(c-1)|A| \geq|A|$, and Hall's Lemma (Lemma 2.1) implies that there is a matching in $H$ that covers all $S \cap X$. By construction of $H$ it has a matching that covers all vertices of $Y$, therefore Lemma 3.1 implies that there exists a matching that covers $S \cap X$ and $Y$, hence it covers $S$. This matching corresponds to an assignment that satisfies all constraints for vertices from $S$, but it is impossible to satisfy the empty clause and we get a contradiction with the fact that $\mu(\square) \leq r$.

The semiadditivity of the measure implies that any resolution proof of the formula $\mathrm{PMP}_{G}$ contains a clause $C$ with the measure in the interval $\frac{r}{2} \leq \mu(C) \leq r$. Let $S \vdash C$ and $|S \cap X|=\mu(C)$. For the sake of brevity let $A=S \cap X$. Since $G$ is a $(r, c)$-boundary expander, $\delta(A) \geq c|A|$. Let $F$ denote the set of edges between $A$ and $\delta(A)$. Every vertex from $\delta(A)$ has exactly one incident edge leading to $A$, therefore $|F|=|\delta(A)|$. Consider one particular edge $f \in F$, let $f=(u, v)$, where $u \in A$. Since $|(S \backslash\{u\}) \cap X|<|S \cap X|$, clause $C$ is not properly implied from the set $S \backslash\{u\}$, i. e. there exists a proper assignment $\sigma$ that satisfies all restrictions in the vertices $S \backslash\{u\}$, but refutes the clause $C$. Such assignment $\sigma$ cannot satisfy the constraint in the vertex $u$, since otherwise $\sigma$ would satisfy $S$ and therefore satisfy $C$. Since $\sigma$ is a proper assignment, $\sigma$ assigns value 0 to all edges that are incident with $u$.

We consider two cases: 1) $\sigma$ refutes a constraint in the vertex $v$;2) $\sigma$ satisfies a constraint in the vertex $v$.

In the first case we consider another assignment $\sigma^{\prime}$ that differs from $\sigma$ in the value of the edge $f$. Note that $\sigma^{\prime}$ is proper and satisfies all constraints from $S$, so it satisfies $C$. Since $\sigma$ does not satisfy $C$, the variable $f$ is contained in $C$.

In the second case $\sigma$ satisfies $v$. There is an edge $e$ incident to $v$ such that $\sigma(e)=1$. The vertex $v$ is a boundary vertex for $A$, therefore the other endpoint of $e$ does not belong to $A$. Consider an assignment $\sigma^{\prime \prime}$ that is obtained from $\sigma$ by changing the values of $f$ and $e, \sigma^{\prime \prime}$ is proper and it satisfies all constraints from $S$, and hence it satisfies $C$. Thus $C$ contains either $e$ or $f$. Thus for all $v \in \delta(A)$ at least one of the edges incident to $v$ occurs in $C$. Therefore the size of the clause $C$ is at least $|\delta(A)| \geq c|A| \geq c r / 2$.

We say that a graph is explicit if it can be constructed in time polynomial in the number of its vertices.

Lemma 3.3 ([IS11], lemma 6.2). For all $d$ large enough and for all $m$ there exists explicit construction of $(r, 0.5 d)$-boundary expander $G(X, Y, E)$ with $|X|=|Y|=m, r=\Omega(m)$ such that degrees of all vertices from $X$ are at most $d$ and degrees of all vertices from $Y$ are at most $d^{2}$.

Corollary 3.1. For all $d$ large enough and for all $C$ and all $n$ and $m \in[n+1, C n]$ there is an explicit construction of $(r, 0.4 d)$-boundary expander $G(X, Y, E)$ with $|X|=m,|Y|=n$ 
and $r=\Omega(n)$ such that degrees of all vertices from $X$ are at most $d$ and degrees of all vertices from $Y$ are at most $d^{2}$.

Proof. The required graph can be obtained from Lemma 3.3 by deleting several vertices from the part $Y$.

Proof of Theorem 3.1. Consider some $d>5$ that satisfies Corollary 3.1; consider $(r, 0.4 d)$ boundary expander $H$ from the Corollary 3.1 that has $m$ and $n$ vertices in parts. Let graph $G$ be obtained from $H$ by adding any matching that covers all vertices from the part $Y$. Graph $G$ is a $(r, c-1)$-boundary expander, since the addition of a matching increases degrees of vertices in $X$ at most by 1 and for every $A \subseteq X$ the size of $\delta(A)$ decreases by at most $|A|$.

Lemma 3.2 implies that the width of any resolution proof of $\mathrm{PMP}_{G}$ is at least $\Omega(n)$. Theorem 2.1 implies that the size of any resolution proof of $\mathrm{PMP}_{G}$ is at least $2^{\Omega(n)}$.

\section{Subgraph extraction}

Let $G(V, E)$ be an undirected graph and $h$ be a function $V \rightarrow \mathbb{N}$ such that for every vertex $v \in V, h(v)$ is at most the degree of $v$. We consider formula $\Psi_{G}^{(h)}$; its variables corresponds to edges of $G . \Psi_{G}^{(h)}$ is a conjunction of the following statements: for every $v \in V$ exactly $h(v)$ edges that are incident to $v$ have value 1 . Formula $\mathrm{PMP}_{G}$ is a particular case of $\Psi_{G}^{(h)}$ for $h \equiv 1$.

Lemma 4.1. For all $d \in \mathbb{N}$ and for all $n$ large enough for any set $V$ of cardinality $n$ and any function $h: V \rightarrow\{1,2, \ldots, d\}$ there exists explicit construction of a graph $G(V, E)$ with the following properties: 1) $V$ consists of two disjoint sets $U$ and $T$ with no edges between them; 2)The degree of every vertex $u \in U$ equals $h(u)-1$ and the degree of every vertex $v \in T$ equals $h(v) ; 3)|U| \geq \frac{n}{2}-2 d^{2}$.

Proof. Let $n \geq 4 d^{2}$ and the vertices $v_{1}, v_{2}, \ldots, v_{n}$ be arranged in non-decreasing order of $h\left(v_{i}\right)$. Let $k$ be the largest number that satisfies the inequality $\sum_{i=1}^{k}\left(h\left(v_{i}\right)-1\right)<$ $\sum_{i=k+1}^{n} h\left(v_{i}\right)-d(d-1)$. We denote $U=\left\{v_{1}, v_{2}, \ldots, v_{k}\right\}$ and $T=V \backslash U$. Obviously, $|U|=k \geq n / 2-d(d-1)$. Now we construct a graph $G$ based on the set of vertices $V$. We start with an empty graph and will add edges one by one. For every vertex $v \in T$ we call co-degree of $v$ the difference between $h(v)$ and the current degree of $v$. From every $u \in U$ we add $h(u)-1$ edges to $G$ that lead from $u$ to distinct vertices of $V \backslash U$. Doing so, we maintain degrees of all $v \in T$ under the value $h(v)$. This always can be done since by the construction of $U$ the total co-degree of all vertices from $T$ is greater than $d(d-1)$, hence for all big enough $n$ there exists at least $d$ vertices with co-degree at least 1 .

While the number of vertices in $T$ with positive co-degree is greater than $d$, we will choose one of those vertices $w \in T$ and add to graph exactly co-degree of $w$ edges that connect $w$ with other vertices from $T$. Finally we have that $T$ contains at most $d$ vertices with co-degrees at most $d$. Now we connect them with distinct vertices from the set $U$, remove that vertices from $U$ and add them to $T$. It is possible that in the last step some vertex $v \in T$ is already connected with several vertices from $U$, in that case we should connect $v$ with new vertices. By this operation we deleted at most $d^{2}$ vertices from $U$ and therefore $|U| \geq n / 2-2 d^{2}$. 
Theorem 4.1. For all $d \in \mathbb{N}$ there is such $D \in \mathbb{N}$ that for all $n$ large enough and for any function $h: V \rightarrow\{1,2, \ldots, d\}$, where $V$ is a set of cardinality $n$, there exists such explicit graph $G(V, E)$ with maximum degree at most $D$, that formula $\Psi_{G}^{(h)}$ is unsatisfiable and the size of any resolution proof for $\Psi_{G}^{(h)}$ is at least $2^{\Omega(n)}$.

Proof. By Lemma 4.1 we construct a graph $G_{1}\left(V, E_{1}\right)$ and a set $U \subseteq V$ of size at least $\frac{n}{2}-2 d^{2}$ such that for all $v \in U$, the degree of $v$ is equal to $h(v)-1$ and for all $v \in V \backslash U$ the degree of $v$ is equal to $h(v)$. Consider graph $G\left(U, E_{2}\right)$ from Theorem 3.1 with $U$ as the set of its vertices. Define a new graph $G(V, E)$, where the set of edges $E$ equals $E_{1} \cup E_{2}$. Recall that edges from the set $E_{2}$ connect vertices of the set $U$ and edges from $E_{1}$ do not connect pairs of vertices from $U$ (that follows from the construction of the graph in Lemma 4.1 ).

For every vertex $v \in V \backslash U$ its degree equals $h(v)$. Therefore if $\Psi_{G}^{(h)}$ is satisfiable, then in any satisfying assignment of $\Psi_{G}^{(h)}$ all edges that are incident to vertices $V \backslash U$ must have the value 1 . After substitution the value 1 for all these variables $\Psi_{G}^{(h)}$ becomes equal to the formula $\mathrm{PMP}_{G_{2}}$ that is unsatisfiable because of Theorem 3.1.

Formula $\mathrm{PMP}_{G_{2}}$ is obtained from $\Psi_{G}^{(h)}$ by substitution of several variables, thus Lemma 2.2 implies that the size of any resolution proof of $\Psi_{G}^{(h)}$ is at least the size of the minimal proof for $\mathrm{PMP}_{G}$, that is at least $2^{\Omega(n)}$ by Theorem 3.1.

\subsection{Colloraries}

Tseitin formulas. A Tseitin formula $T_{G}^{(f)}$ can be constructed by an arbitrary graph $G(V, E)$ and a function $f: V \rightarrow\{0,1\}$; variables of $T_{G}^{(f)}$ corresponds to edges of $G$. The formula $T_{G}^{(f)}$ is a conjunction of the following conditions: for every vertex $v$ we write down a CNF condition that encode that the parity of the number of edges incident to $v$ that have value 1 is the same as the parity of $f(v)$.

Based on the function $f: V \rightarrow\{0,1\}$ we define a function $h: V \rightarrow\{1,2\}$ by the following way: $h(v)=2-f(v)$. In other words if $f(v)=1$, then $h(v)=1$, and if $f(v)=0$, then $h(v)=2$. By Theorem 4.1 there exists such number $D$, that for all $n$ large enough it is possible to construct graph $G$ with $n$ vertices of degree at most $D$ such that the size of any resolution proof of the formula $\Psi_{G}^{h}$ is at least $2^{\Omega(n)}$.

Note that every condition corresponding to a vertex of the formula $T_{G}^{(h)}$ is implied from the condition corresponding to the formula $\Psi_{G}^{h}$. Since the resolution proof system is implication complete, every condition of $T_{G}^{(h)}$ may be derived from a condition of $\Psi_{G}^{h}$ by derivation of size at most $2^{D}$ Hence all clauses of the Tseitin formula may be obtained from clauses of formula $\Psi_{G}^{h}$ by the derivation of size $O(n)$. Thus the size of any resolution proof of $T_{G}^{(f)}$ is at least $2^{\Omega(n)}$. This lower bound was proved in the paper [Urq87].

Complete graph. Let $K_{n}$ be a complete graph with $n$ vertices and $h: V \rightarrow$ $\{0,1, \ldots, d\}$, where $d$ is a some constant. Let formula $\Psi_{K_{n}}^{(h)}$ be unsatisfiable. By Theorem 4.1 there exists $D$ such that for all $n$ large enough there exists an explicit graph $G$ with $n$ vertices of degree at most $D$ that the size of any resolution proof of $\Psi_{G}^{h}$ is at least $2^{\Omega(n)}$. The graph $G$ can be obtained from $K_{n}$ by removing of several edges, hence the formula $\Psi_{G}^{(h)}$ can be obtained from $\Psi_{K_{n}}^{(h)}$ by the substitution zeroes to edges that do not 
present in $G$. Therefore by Lemma 2.2 the size of the resolution proof of $\Psi_{K_{n}}^{(h)}$ is at least $2^{\Omega(n)}$.

\section{Acknowledgements}

The authors are grateful to Vsevolod Oparin for fruitfull discussions.

\section{References}

[Ale04] Michael Alekhnovich. Mutilated chessboard problem is exponentially hard for resolution. Theor. Comput. Sci., 310(1-3):513-525, January 2004.

[BSW01] E. Ben-Sasson and A. Wigderson. Short proofs are narrow - resolution made simple. Journal of ACM, 48(2):149-169, 2001.

[DR01] Stefan S. Dantchev and Søren Riis. "planar" tautologies hard for resolution. In FOCS, pages 220-229, 2001.

[Hak85] Armin Haken. The intractability of resolution. Theoretical Computer Science, 39:297-308, 1985.

[HLW06] S. Hoory, N. Linial, and A. Wigderson. Expander graphs and their applications. Bulletin of the American Mathematical Society, 43:439-561, 2006.

[IS11] Dmitry Itsykson and Dmitry Sokolov. Lower bounds for myopic DPLL algorithms with a cut heuristic. In Proceedings of the 22nd international conference on Algorithms and Computation, ISAAC'11, pages 464-473, Berlin, Heidelberg, 2011. Springer-Verlag, available as ECCC Report TR12-141.

[MCW02] S. Vadhan M. Capalbo, O. Reingold and A. Wigderson. Randomness conductors and constant-degree expansion beyond the degree/2 barrier. In Proceedings of the 34th Annual ACM Symposium on Theory of Computing, pages 659-668, 2002.

[Raz01a] Ran Raz. Resolution lower bounds for the weak pigeonhole principle. Technical Report 01-021, Electronic Colloquium on Computational Complexity, 2001.

[Raz01b] Alexander A. Razborov. Resolution lower bounds for the weak pigeonhole principle. Technical Report 01-055, Electronic Colloquium on Computational Complexity, 2001.

[Raz03] Alexander A. Razborov. Resolution lower bounds for the weak functional pigeonhole principle. Theoretical Computer Science, 303(1):233-243, 2003.

[Raz04] Alexander A. Razborov. Resolution lower bounds for perfect matching principles. Journal of Computer and System Sciences, 69(1):3-27, 2004. 
[SB97] T. Pitassi S. Buss. Resolution and the weak pigeonhole principle. In Proceedings of the CSL97, Lecture Notes in Computer Science, volume 1414, page 149-156, 1997.

[Urq87] A. Urquhart. Hard examples for resolution. JACM, 34(1):209-219, 1987.

[Urq03] Alasdair Urquhart. Resolution proofs of matching principles. Annals of Mathematics and Artificial Intelligence, 37(3):241-250, March 2003. 